The evolving sense of agency:

\section{Context recency and quality modulate the interaction between prospective and retrospective processes}

\author{
Simone Di Plinio1*, Simone Arnò², Mauro Gianni Perrucci,3, \\ Sjoerd J.H. Ebisch 1,3
}

1 Department of Neuroscience Imaging and Clinical Sciences, G.

D'Annunzio University of Chieti-Pescara, Chieti 66100, Italy;

2 Department of Psychological Sciences, G d'Annunzio University of Chieti-Pescara, Chieti, Italy;

3 Institute for Advanced Biomedical Technologies (ITAB), G

d'Annunzio University of Chieti-Pescara, Chieti, Italy;

* Corresponding author. Address: Department of Neuroscience Imaging and Clinical Sciences, G. d'Annunzio University of Chieti-Pescara, Chieti 66100, Italy. E-mail: simone.diplinio@unich.it

Conflict of Interest: The authors declare no competing financial interests.

Running Head: Interaction among agency subprocesses

Keywords: Intentional binding; Sense of Agency; Psychosis;

Sensorimotor learning; Implicit learning; Perception.

Funding: This work was supported by the BIAL Foundation grant number 195/16 to SE, and by the "Departments of Excellence 2018-2022" initiative of the Italian Ministry of Education, Research, and University for the Department of Neuroscience, Imaging and Clinical Sciences (DNISC) of the University of Chieti-Pescara. 
The evolving sense of agency: Context recency and quality modulate the interaction between prospective and retrospective processes

\section{ABSTRACT}

2 Humans acquire a sense of agency through their interactions with

3 the world and their sensory consequences. Previous studies have

4 highlighted stable agency-related phenomena like intentional

5 binding, which depend on both prospective, context-dependent and

6 retrospective, outcome-dependent processes. In the current

7 study, we investigated the interaction between prospective and

8 retrospective processes underlying the adaptation of an ongoing

9 sense of agency. The results showed that prospective intentional

10 binding developed during a temporal window of up to 20 prior

11 events were independent of the nature of the ongoing event. By

12 contrast, the characteristics of the ongoing event

13 retrospectively influenced prospective intentional binding

14 developed during a temporal window narrower than 6 prior events.

15 These findings characterize the interaction between prospective 16 and retrospective mechanisms as a fundamental process to

17 continuously update the sense of agency through sensorimotor

18 learning. High psychosis-like experience traits weakened this

19 interaction, suggesting that reduced adaption to the context

20 contribute to altered self-experience. 
The evolving sense of agency: Context recency and quality modulate the interaction between prospective and retrospective processes

\section{1. INTRODUCTION}

2 The sense of agency is the feeling of controlling the initiation and 3 execution of our actions and of assimilating their consequences 4 thereof. There is a consensus that humans acquire a sense of agency 5 through their interactions with the environment and particularly 6 through the sensory feedback of performed actions. The relevance of 7 understanding the many facets of the sense of agency is underlined by 8 both its importance for healthy development (Synofzik et al., 2013; 9 Haggard, 2017; Bandura, 2018) and its disruption in psychopathology 10 (Hauser et al., 2011; Moore \& Fletcher, 2012; Moore, 2016).

A commonly used approach to investigating the sense of agency 12 and its alterations is the exposure of participants to experimental 13 conditions that differ in the predictability of sensory feedback 14 (Haggard et al., 2002), in the temporal proximity between actions and consequences (van Kemenade et al., 2018; Wen, 2019), or in the subjective degree of control induced in the participant (Kumar \& Srinivasan, 2013). During the procedure, implicit or explicit ratings of agency are collected in a series of events and are often averaged within specific conditions (Haggard, 2017; Imaizumi \& Tanno, 2019).

Some implicit ratings of agency-related perceptual phenomena have been described as stable, unbiased, and replicable measures of the sense of agency. For instance, intentional binding (Haggard et al., 2002; Voss et al., 2010; Berberian et al., 2012; Bohrani et al., 2017) is the subjective shift of the perceived timing of an action toward its consequence, or vice versa. Importantly, intentional binding effects can indicate a prospective or retrospective component of the sense of agency. A prospective component is based on a contextual and probabilistic event coding and is usually indicated by increased intentional binding in a context of high probability of action feedback, compared to a context of low probability. By contrast, a retrospective sense of agency concerns outcome-dependent,

32 a posteriori inferences about the action feedback (Moore \& Haggard, 2008; Synofzik et al., 2008, 2013).

Previous findings suggest that the sense of agency has a fundamental flexibility that conceivably arises from the online 
The evolving sense of agency: Context recency and quality modulate the interaction between prospective and retrospective processes

1 adaptation of sensorimotor predictions during ongoing behavior (Clark,

2 2013). By mediating the development of action awareness, implicit

3 sensorimotor learning may be a fundamental process in establishing

4 the sense of agency (McDougle et al., 2016; Kim et al., 2019). Implicit

5 learning is an endogenous process involved in generating and updating

6 sensorimotor predictions in the brain (Friston \& Kiebel, 2009; Wolpert

7 et al., 2011; Legaspi \& Toyoizumi, 2019; Di Plinio, Perrucci, \& Ebisch,

8 submitted) that affects behavioral temporal estimates (Taylor et al.,

9 2014; Stark-Inbar et al., 2017). Therefore, implicit learning

10 plausibly supports a continuous, trial-after-trial update of the sense

11 of agency. Given that the context-based (prospective) sense of agency

12 is based on the implicit learning of the consistency of the outcome-

13 based (retrospective) element, we argue that prospective and

14 retrospective mechanisms of the sense of agency are not independent;

15 we propose that they may interact to continuously update the context

16 generated by the quality of the sensory action outcome. However, the

17 way in which prospective and retrospective mechanisms interact to 18 continuously update the ongoing sense of agency is still poorly 19 understood.

Especially regarding the prospective sense of agency, 21 experimental paradigms typically assume that a given context is 22 developed by the agent within a given run or block of sensorimotor 23 events. However, this assumption depends on some critical premises. 24 The first premise is within-run homogeneity: a run-based approach presumes that events within a given run are homogeneous. To the contrary, studies have reported within-run inhomogeneities (Moore et al., 2011; Moore et al., 2012; Sidarus et al., 2017), suggesting that contexts of increased versus decreased agency can alternate within the same run. Furthermore, although prospective and retrospective sense of agency are both based on the probability of the action outcomes, previous studies have typically distinguished prospective and retrospective sense of agency components without considering their interaction. However, outcomes could either confirm or contradict the agency context defined by prior events, possibly leading to conflicts between the prospective and retrospective components. Such conflicts could constitute relevant information which flexibly update a possibly changing context. Another premise is that there are no cross-run 
The evolving sense of agency: Context recency and quality modulate the interaction between prospective and retrospective processes

1 interactions. This means that events in previous runs are presumed 2 not to affect events in subsequent runs. This is highly improbable, 3 because the perception of outcomes relies on both structural and 4 contextual short-term expectations (Desantis et al., 2016; Dogge et 5 al., 2019). Finally, the cross-participant pseudo-randomization of 6 the run order is thought to avoid the introduction of run-order biases, 7 while effects due to run order could provide additional information. 8 Thus, it is reasonable to expect that critical insights into the 9 constitution of the sense of agency can be achieved by investigating 10 the prospective and retrospective sense of agency as interacting 11 components within a context that is continuously updated through 12 implicit sensorimotor learning.

The present study addressed several questions related to these issues. For example, are retrospective mechanisms based on single trial information, or are they influenced by the context? Similarly, are prospective-contextual mechanisms stable regardless of the nature of the ongoing action event, or do they also depend on the actual action outcome? How many events are necessary to establish a given context of a prospective sense of agency? We further explored the clinical relevance of the interaction between the prospective and retrospective sense of agency components. Because clinical symptoms of psychosis are correlated with an impaired adaptation to ongoing behavior (Frith et al., 2000; Blakemore et al., 2002; Ford \& Mathalon, 2005; Moore \& Fletcher, 2012, Martinelli et al., 2017; Bansal et al., 2019), we investigated how psychosis-relevant personality traits interact with the embodiment of a dynamic sense of agency. Considering previous literature on the association between uncertainty (or ambiguity) and psychosis (Linney et al., 1998; Van Dael et al., 2006; Garety et al., 2011), we hypothesized that there could be an

30 interaction between the prospective and retrospective components which may create ambiguous situations of "conflict" between the expected and actual action outcomes and that this interaction may be relevant for psychosis-relevant traits.

To clarify these questions, and expanding on previous studies, 35 the present study analyzed prospective and retrospective intentional 36 binding effects by considering the entirety of the experiment as a 
The evolving sense of agency: Context recency and quality modulate the interaction between prospective and retrospective processes

1 unique, subjective sequence of events in which the context is

2 constantly updated. This approach was intended to avoid the biases of

3 within-run inhomogeneity and cross-run interactions. We also tested

4 whether intentional binding may change depending on how long a given

5 context is assumed to last (e.g., a few trials vs. many trials). Thus,

6 we investigated the effect of the context at multiple temporal scales.

7 A relatively large number of participants was used to achieve high

8 statistical power on a trial-based approach.

We hypothesized that the interaction between the prospective and retrospective sense of agency could be affected by the number of events considered to form a context. For example, more recent and earlier trials could reflect a "narrow context" and an "extended context," respectively. Whereas previous studies have shown that a context with a high probability of receiving sensory feedback increases the sense of agency, we expected that the percentage of feedback trials within narrow and extended contexts would differently influence the sense of agency. Individual differences in personality traits were investigated starting from the belief that increased psychosis-like experiences may affect individuals' adaptation to the ongoing behavior. Consequently, this aberrant adaptation may perturb the sense of agency measured by intentional binding, specifically when action outcomes conflict with context-based expectancies.

\section{MATERIALS and METHODS}

\subsection{Participants}

The overall sample of this study consisted of 101 non-clinical, 27 healthy Italian adults (48 males and 53 females; age $23 \pm 3$ ). None of

28 the participants had ever suffered from any psychiatric or neurological disorder. All the participants in the study had a normal or corrected-to-normal vision and gave written informed consent before taking part in the study. All the 101 participants completed the psychometric questionnaires included in the study, while a subset of

3361 participants (29 males and 32 females; age $23 \pm 3$ ) was included 34 for the sense of agency task (these include the 20 participants of 35 the positive-control group in Di Plinio et al., 2019a and the 39 
The evolving sense of agency: Context recency and quality modulate the interaction between prospective and retrospective processes

1 participants of the fMRI experiment in Di Plinio, Perrucci, and

2 Ebisch, submitted). A relatively high number of participants was

3 chosen for the study since we wanted to overcome biases related to

4 the pseudo-randomization of runs and to perform trial-based analyses.

5 Since the remaining 40 subjects performed a different version of the

6 sense of agency task (as described in Di Plinio et al. 2019a), these

740 subjects were only used for the factor analysis on psychometric

8 questionnaires.

\subsection{Psychometric questionnaires}

11 In a way analogous to a previous study (Di Plinio et al., 2019a), we 12 investigated the relation between the sense of agency and personality 13 traits with a focus on factors related to psychotic disorders (Asai 14 et al., 2008; Jeannerod, 2009; Asai et al., 2011; Gallagher \& Trigg, 15 2016). The following questionnaires were administered to the overall 16 cohort $(\mathrm{N}=101)$.

The Big Five Questionnaire (BFQ, Caprara et al., 1993), which 18 follows the five-factor model (Costa \& McCrae, 1985) was administered 19 to participants. In the $\mathrm{BFQ}$, the five personality dimensions are defined through sixty items rated from 1 to 5: neuroticism (12 items, $\alpha=0.85)$, extraversion (12 items, $\alpha=0.61)$, conscientiousness (12 items, $\alpha=0.79$ ), agreeableness (12 items, $\alpha=0.77$ ), and openness to experience (12 items, $\alpha=0.73$ ).

The Intolerance of Uncertainty Scale (IUS-12, Carleton et al., 2007) was employed to assess the tolerance of uncertain situations. It consists of 12 items rated on a 5-points scale, for which two subscales are identified: inhibitory uncertainty (7 items, $\alpha=0.73$ ) and prospective uncertainty (5 items, $\alpha=0.81$ ).

The Community Assessment of Psychic Experience (CAPE, Konings et

30 al., 2006) was used to assess the presence of psychosis-like in the general population through its three subscales: positive symptoms (20 items, $\alpha=0.79)$, negative symptoms (14 items, $\alpha=0.82)$, and depressive symptoms ( 8 items, $\alpha=0.74$ ). 
The evolving sense of agency: Context recency and quality modulate the interaction between prospective and retrospective processes

The Schizotypal Personality Questionnaire (SPQ, Raine, 1991;

Fossati et al., 2003) consists of 74 questions to which the participants may answer "yes" (=1) or "no (=0). The three subscales, 4 which are made up by the sum of positive answers, are: cognitiveperceptual (25 items, $\alpha=0.84$ ), interpersonal (33 items, $\alpha=0.85$ ), and disorganized (16 items, $\alpha=0.86$ ).

Finally, the State-Trait Anxiety Inventory (STAI2, Spielberger et al., 1983) is a 20-items scale. The participants are asked to rate each item from one to four. The final score is obtained by the algebraic sum of the items $(\alpha=0.87)$.

\subsection{Behavioral procedure}

The participants performed a sense of agency paradigm used also in previous studies (Haggard et al., 2002; Voss et al, 2010; Di Plinio et al., 2019a; Di Plinio et al., submitted). On each trial, the participants performed voluntary keypresses with the right index finger while watching a clock hand rotating on a screen. The clock was labeled with numbers positioned at 5-minutes intervals on a standard circular clock. The participants' task was to report the timing of their keypress. In other words, the participants reported the clock time indicated by the clock hand each time they pressed the key. The clock hand rotation began at a random position in each trial and performed a full rotation every $2560 \mathrm{~ms}$. The inter-trial interval was jittered between two and three seconds.

To avoid behavioral biases, the participants were instructed to avoid the planning of their keypresses, to avoid answering in a stereotyped way, and to avoid answering before the end of the first clock rotation. Each participant performed a brief training session before the experiment. The behavioral responses were collected using the E-Prime software (Schneider et al., 2012) while participants were sitting $60 \mathrm{~cm}$ from the 20-inches monitor used for the presentation of the stimuli. Sensory feedback used in the present study were acoustic tones. Each tone lasted $200 \mathrm{~ms}$ and consisted of a single, continuous pulse at $1000 \mathrm{~Hz}$ presented binaurally $250 \mathrm{~ms}$ after the keypress. Trials in which the tone was presented were labeled "feedback trials", 
The evolving sense of agency: Context recency and quality modulate the interaction between prospective and retrospective processes

1 while trials in which the tone was not presented were labeled "no-

2 feedback trials".

Each participant performed five experimental runs in which intentional binding was modulated through the rate of sensory feedback. In two of these runs, there was a probability of 50\% for feedback trials: half of the total trials were feedback trials and the remaining half were no-feedback trials. In two other runs, 75\% of the trials were feedback trials and the remaining 25\% were no-feedback trials. Finally, in one run, there were only no-feedback trials. Each run consisted of 32 trials, for a total of 160 trials. Run order was pseudo-randomized across participants, and trial order was pseudorandomized within each run. Between-run intervals, during which the subject was told to rest, lasted for an average of 28 seconds. The whole procedure lasted approximately 45 minutes for each participant. The task is illustrated in Figure 1.

The perceptual shift ( $\Delta$, expressed in milliseconds), namely the difference between the real and the perceived keypress time, was measured for each trial. To note, the perceptual shift and the phenomenon of intentional binding should not be confused. While the former represents the difference between real and perceived keypress time, the latter is a phenomenon that refers to the subjective compression of the time interval between an action and its consequence.

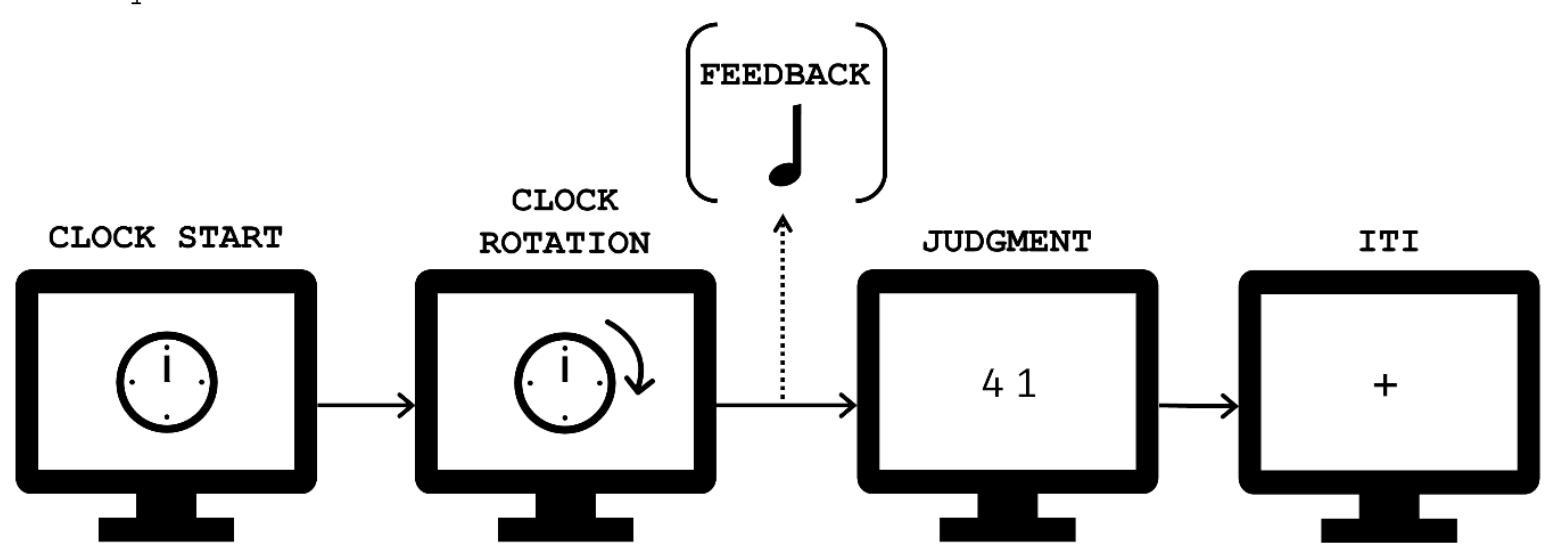

Figure 1. Behavioral task. The participants performed self-decided keypresses with the right index finger while watching a clock hand rotating on a screen. The participants were instructed to judge the clock time when they pressed the key. Auditory feedback (or nothing, contingent on the current trial) was binaurally presented through headphones only in feedback trials, and not in 
The evolving sense of agency: Context recency and quality modulate the interaction between prospective and retrospective processes

1 no-feedback trials. The participants started each trial by pressing a button

2 with the left index finger.

\subsection{Statistical analysis}

5

6

7

8

9

10

11

12

2.4.1. Defining the variable "context"

Our aim was to assess the link between an implicit sense of agency scores (intentional binding calculated through perceptual shifts, $\Delta$ ) and a temporally dynamic context. Thus, considering the experiment as a unique sequence of feedback and no-feedback trials, we estimated a regressor (parametric modulator), namely context, which indicated the proportion of feedback trials in a window of $k$ trials before the ongoing trial. In each participant, for each trial $t$, we calculated the context as

$$
\mathrm{C}_{\mathrm{k}, \mathrm{t}}=\frac{\mathrm{F}_{\mathrm{k}, \mathrm{t}}-N \mathrm{~F}_{\mathrm{k}, \mathrm{t}}}{\left(\mathrm{F}_{\mathrm{k}, \mathrm{t}}+N \mathrm{~F}_{\mathrm{k}, \mathrm{t}}\right)}
$$

where $\mathrm{C}_{\mathrm{k}, \mathrm{t}}$ is the value of the context (standardized proportion of feedback trials before the current trial) for the current trial t; $F_{k, t}$ is the number of feedback trials among the $k$ trials antecedent the current trial $t$; and $\mathrm{NF}_{k, t}$ is the number of no-feedback trials among the $k$ trials antecedent the current trial $t$. Obviously, the first $k$ trials of the experiment were excluded due to the impossibility to have an associated context. Since we wanted to investigate the dynamic effect of the context on intentional binding on multiple temporal scales, values of $k$ were varied in the interval [1-20], with unitary steps. Values of $\mathrm{C}$ within each $k$ and within each participant were standardized before further analyses.

2.4.2. Effect of the context on the perceptual shift

Instead of averaging $\Delta$ within each block, we performed a trialbased analysis by implementing a mixed-effects multilevel regression model. Within the model, the following variables were considered: the dependent variable was the perceptual shift $(\Delta)$; the categorical factor trial-type represented the type of trial and therefore could be equal to 'feedback' or 'no-feedback'; the continuous regressor 
The evolving sense of agency: Context recency and quality modulate the interaction between prospective and retrospective processes

1 context was a numerical factor representing the context as the

2 standardized proportion of feedback trials before each trial as

3 described in $\$ 2.4 .1$; finally, subject was added as a random grouping

4 factor representing each participant. For each level of the grouping

5 factor subject (i.e., for each participant) both a random intercept

6 and random slopes were added to allow the extraction of single-subject

7 estimates (best linear unbiased predictors). A separate model was run

8 for each trial window, that is, for each value of $k$.

It can be pointed out that a trial-based investigation could suffer from the effects of implicit sensorimotor learning during the task (Taylor et al., 2014; Stark-Inbar et al., 2017). To consider this possible confound, we added a covariate, named linear trend, in the model. This covariate represented normalized trial numbers (with a progressive linear increase from 1 to $N$, where $N$ is the total number of valid trials) and thus accounted for linear trends over time in the perceptual shift $(\Delta)$. Another bias in this type of analysis can derive from the presence of outliers in the data. To prevent this issue, for each participant, outlier trials were detected and removed when they differed three scaled median absolute deviations from the median . agency on a trial-based approach were investigated following a factor analysis with orthomax rotation using the fourteen original psychometric scales (see \$2.2) to obtain orthogonal trait factors (see also Di Plinio et al, 2019a/b). A parallel analysis (Horn, 1965; Ledesma \& Valero-Mora, 2007) was implemented to select the number of factors to retain: 10,000 simulated eigenvalues were generated using a Monte Carlo procedure which started from random datasets with equal structure as the original dataset. Then, the number of factors to retain in the factor analysis was selected as the number of eigenvalues above the $95^{\text {th }}$ percentile of the null distribution.

The appropriateness of the factor analysis was tested using a Monte Carlo measurement (1,000 cycles) of Tucker's congruence 
The evolving sense of agency: Context recency and quality modulate the interaction between prospective and retrospective processes

1 coefficient (Korth \& Tucker, 1976; Lorenzo-Seva \& ten Berge, 2006; de

2 Winter et al., 2009). Within each cycle, the overall sample of 101

3 participants was randomly split into two subsamples. Tucker's

4 congruence coefficient $(K)$ between the two subsets of factors was

5 estimated in each cycle, and the procedure was repeated using

6 different sample sizes $(\mathrm{N} 1=\mathrm{N} 2=[20,25,30,35,40,45,50])$.

7 Average values of $\mathrm{K}$ were calculated, and a hyperbolic trend was fitted

8 to estimate the predicted $\mathrm{K}$ based on our overall sample size. binding

The associations between personality traits and the sense of agency were investigated after extracting random statistics from the mixed-effects model described in section \$2.4.2. For each possible effect of interest (i.e., trial-type, context, and the interaction context:trial-type), the best linear unbiased predictor (BLUP) was extracted from each subject and then the group effect was added to obtain individual estimates of the effect $\left(\beta_{T}, \beta_{C}, \beta_{C^{*} T}\right)$. This procedure was repeated for each trial window $k$.

Subsequently, a multivariate regression model was run for each effect of interest. The dependent variable was represented by the individual estimates in the 20 different trial windows. The five psychometric factors resulting from the factor analysis (F1-5) were used as continuous regressors. To note, since the orthomax rotation was used to obtain orthogonal trait factors, these analyses do not entail collinearity issues. Results were corrected for multiple comparisons using false discovery rate.

\section{RESULTS}

\subsection{Effect of the context on the perceptual shifts}

The results and statistics of the trial-based analysis are reported

32 in Figure 2 and Table I. The main effect of trial-type, indicating

33 the retrospective component, was significant across all the trial 34 windows explored (Figure 2a). Instead, the main effect of the context 
The evolving sense of agency: Context recency and quality modulate the interaction between prospective and retrospective processes

1 alone, reflecting a prospective component, was significant when the 2 context was estimated by using a trial window of 7 or more prior 3 trials (Figure 2b). There also was a significant interaction between 4 context and trial-type (Figure 2c) that changed dynamically depending 5 on the size of the trial window. Specifically, context and trial type 6 interacted when the context was estimated using trial windows 7 comprised between 1 to 6 trials. This interaction was not significant 8 anymore when the context was estimated using trial windows comprised 9 between 7 to 20 trials. Residuals were normally distributed and there were no differences between residuals from feedback vs. no-feedback trials as reported in Figure $2 \mathrm{~d}$.
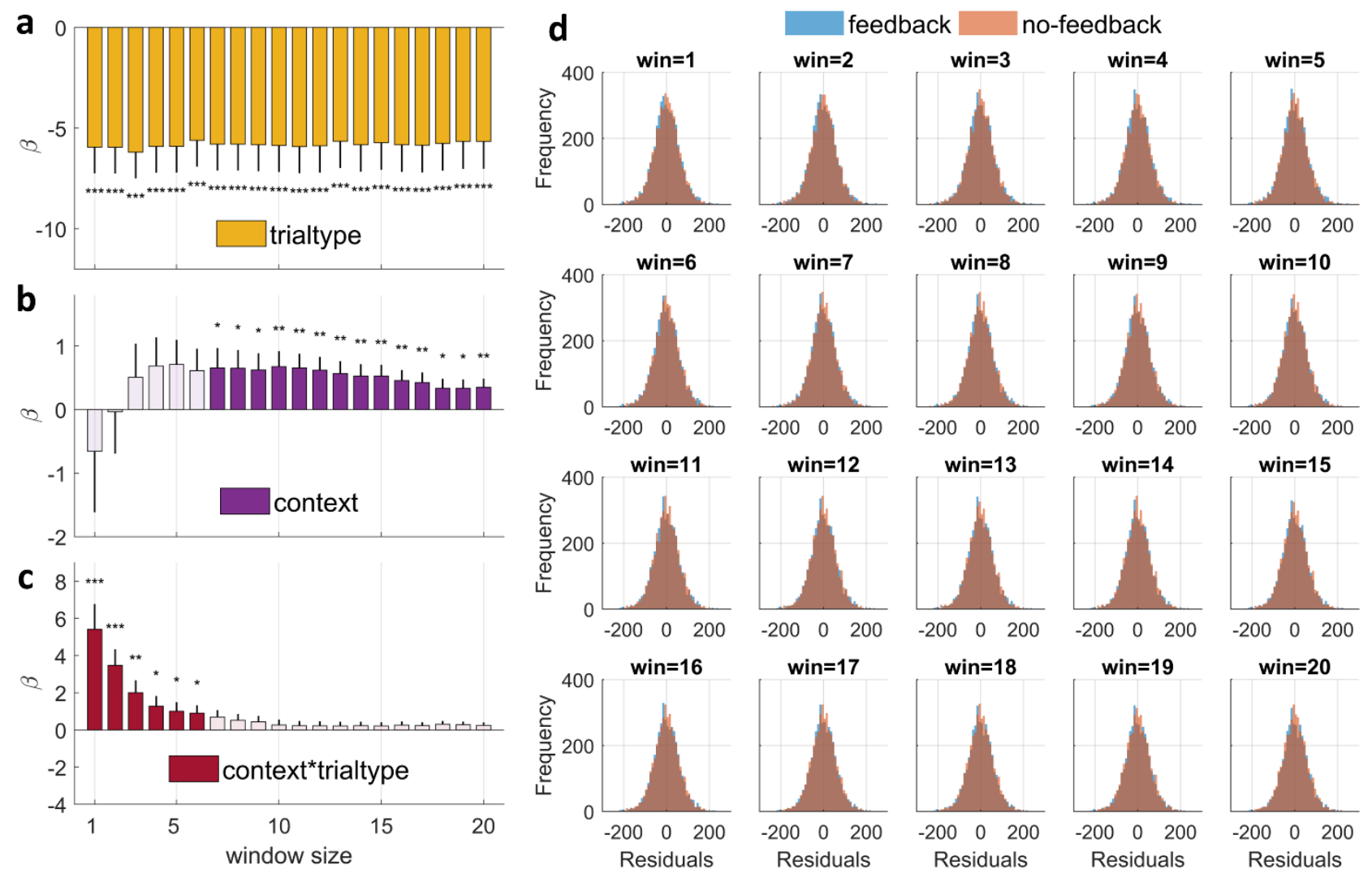
standard errors for the effect of trial-type (a), context (b), and for the interaction context:trial-type (c) derived from multilevel regression models. Significant results are indicated by more intense color bars and asterisks (***: $\left.\mathrm{p}<0.001 ;{ }^{* *}: \mathrm{p}<0.01 ;{ }^{*}: \mathrm{p}<0.05\right)$. (d) Residuals for each regression model. Residuals (in milliseconds) related to feedback and no-feedback trials are reported in blue and red colors, respectively. The substantial overlap is evident. 
The evolving sense of agency: Context recency and quality modulate the interaction between prospective and retrospective processes

The essence of the interaction between context and trial-type is illustrated in Figure 3, in which predictions relative to feedback 3 and no-feedback trials with respect to the context are illustrated 4 for increasing trial window lengths. In the case of no-feedback trials and with a narrower trial window size (orange colors in Figure 3a), 6 individuals shifted their perception towards the expected temporal 7 position of the tone within contexts of high-tone probability.
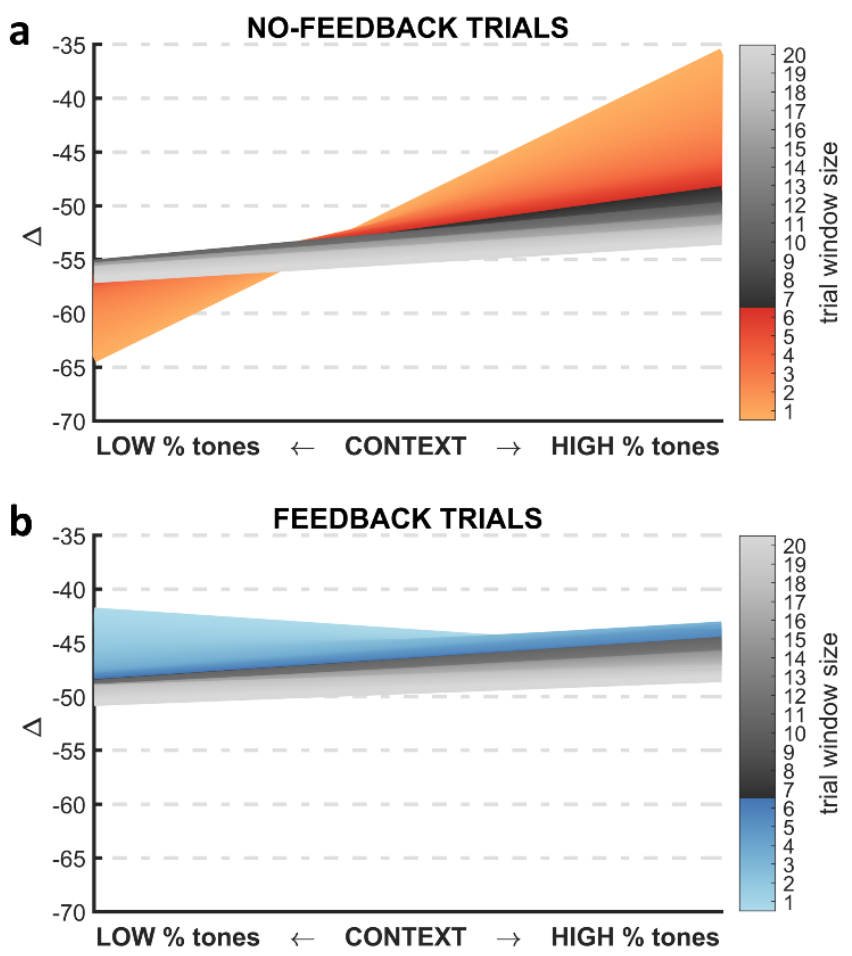

8 Figure 3. Interaction context:trial-type. The figure displays predictions of

9 the perceptual shift in no-feedback trials (a) and feedback trials (b), using 10 varying levels of context and trial windows. A narrow context (calculated using 1-6 trials) had a large positive effect on no-feedback trials and no effect (or a slightly negative effect) on feedback trials, reflecting the significance of the interaction context:trial-type. Instead, an extended context (estimated using 7-20 trials) had a moderate, positive effect on both no-feedback and feedback trials (grey lines in both subfigures), reflecting the significance of the factor context.

Thus, when considering narrow trial windows, there was a strong effect of the context on the perceptual shifts for the no-feedback trials. Instead, this trend was no longer observed for the same trial window lengths during the feedback trials (blue colors in Figure 3b). In fact, the low versus high probability of action feedback had almost no impact on intentional binding in feedback trials. On the other 
The evolving sense of agency: Context recency and quality modulate the interaction between prospective and retrospective processes

1 hand, when the context was estimated using extended trial windows

2 (i.e., 7-20 trials), the context showed a moderate positive 3 association with the perceptual shifts in both feedback and no4 feedback trials, without distinction (grey lines in Figure 3a-b).

\section{$6 \quad 3.2$. Trait factor results}

7 The parallel analysis indicated five factors to use, as this was the 8 number of eigenvalues above the 95th percentile from the null 9 distribution (Figure 4a; see also Di Plinio et al., 2019a/b). The 10 Monte Carlo procedure for computing Tucker's congruence coefficient 11 highlighted a high consistency for the factor analysis (K 0.95, Figure 12 4b). The score distributions of the five factors extracted in this 13 way are reported in Figure 4c. Average scores, standard deviations, 14 and ranges for all original subscales are reported in Table II. The 15 following subscales had the highest loadings: (F1) STAI2, IUS16 inhibitory, CAPE-depression, BFI-neuroticism (negative affect trait); 17 (F2) SPQ-cognitive-perceptual, CAPE-positive, CAPE-negative 18 (psychosis-like experiences trait); (F3) IUS-prospective, BFI19 extraversion, BFI-agreeableness, BFI-openness (positive sociality 20 trait); (F4) SPQ-interpersonal, SPQ-disorganization (schizotypal trait); (F5) BFI-conscientiousness. 

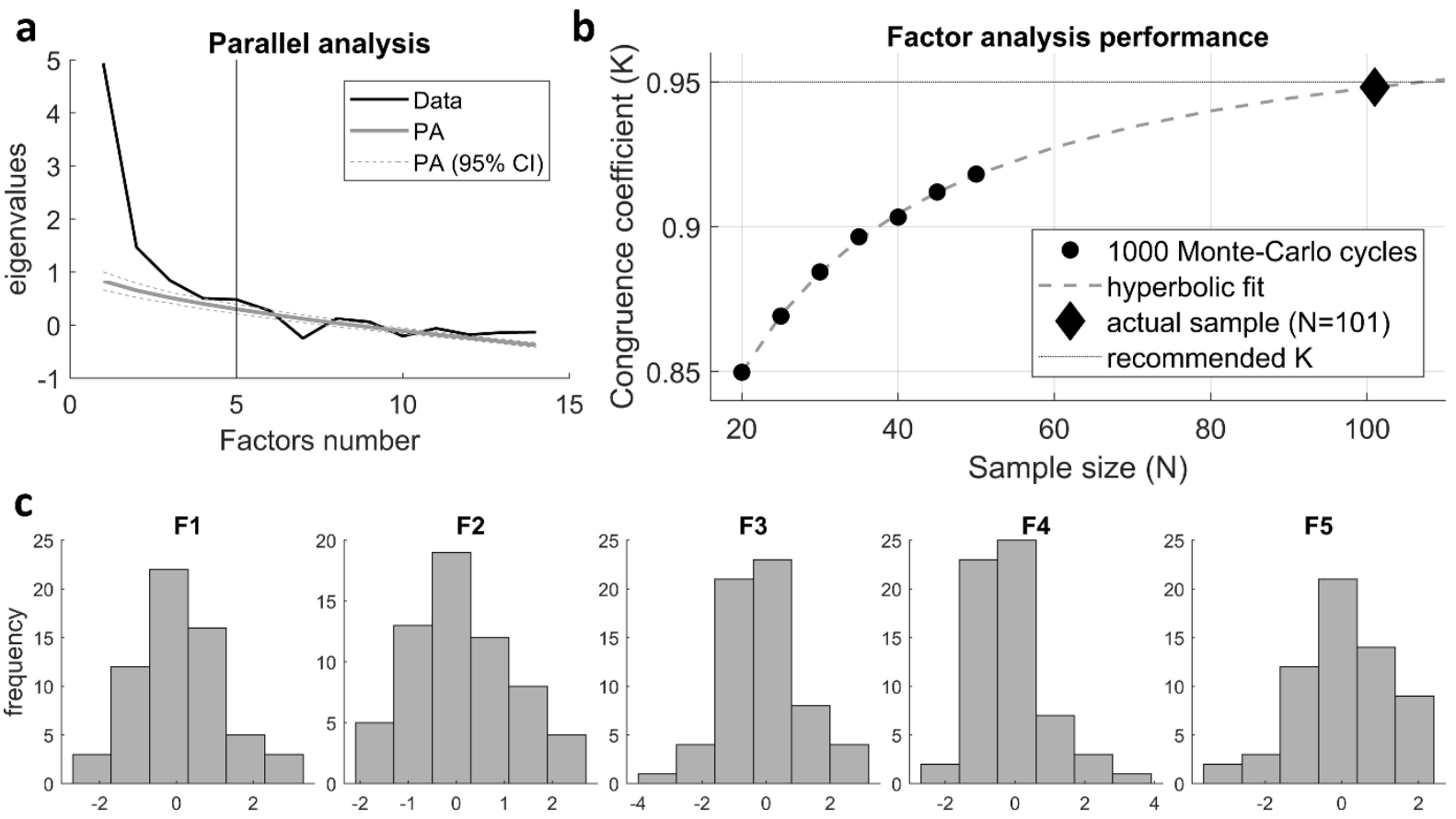

Figure 4. Factor analysis. (a) Eigenvalues distributions from the parallel analysis indicated five factors to retain in the factor analysis. (b) The analysis of Tucker's congruence coefficient suggested a high consistency of the factor analysis. (c) The distribution of scores across the 61 subjects used in this study is shown for each factor.

\subsection{Relationships between sense of agency and psychometric factors}

Associations between the five psychometric factors and the three effects of the multilevel regression models (trial-type, context, context:trial-type) were evaluated using multivariate models. Only one effect was significant after the correction for multiple comparisons: the psychometric factor F2 (related to psychosis-like experiences) was negatively associated with the strength of the interaction context:trial-type, especially when the trial windows for the estimation of the context were comprised between 1 and 6 trials $\left(F_{(19)}=3.1 ; p=9.1 e-06 ;\right.$ multivariate model $)$. Figure 5 reports this result, showing that the context:trial-type interaction in a narrow context (trial window $<6$ prior events) was strong in individuals with low scores in psychosis-like experience traits, but was weaker with higher scores in these traits. 
The evolving sense of agency: Context recency and quality modulate the interaction between prospective and retrospective processes

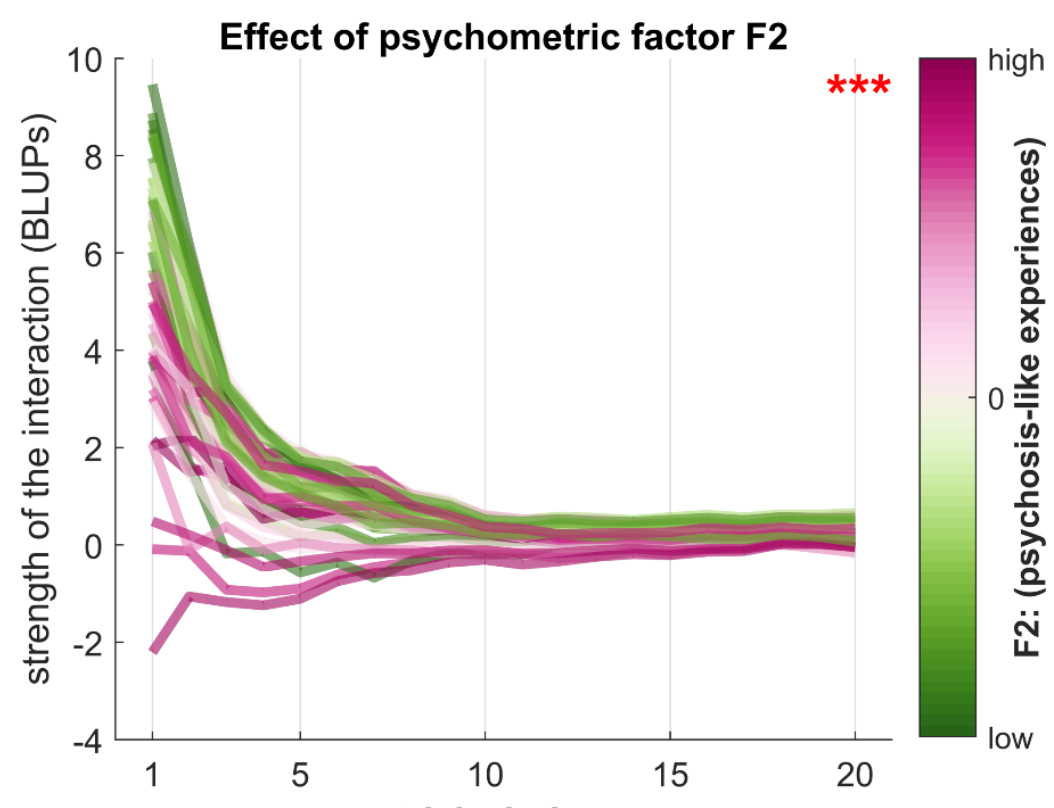

1

Figure 5. Effect of the factor F2 on perception. The figure displays the significant result among multivariate models after corrections for multiple comparisons. Green and purple colors indicate individuals with low and high scores in F2, respectively. As shown in the figure, low scorers in F2 exhibit a strong context:trial-type interaction with a narrow trial window comprised between 1 and 6 trials (green colors), while this interaction tends to become irrelevant in high scorers (purple colors; $\star * *: p=9.1 e-06$ ).

\section{DISCUSSION}

The present study investigated the prospective and retrospective components of the implicit sense of agency using multiple temporal scales to define the context and implementing a trial-based approach. In particular, we defined the context as the proportion of feedback trials preceding each trial. Our findings showed that an extended, less recent context (considering 7 to 20 trials prior to the actual trial) had a moderate effect on the prospective sense of agency. This contextual effect was independent of trial type, that is, the presence or absence of the sensory feedback. By contrast, a narrow, more recent context (1 to 6 trials prior to the actual trial) had a strong effect on the prospective sense of agency only when the trial type conflicted with the context, that is, when no-feedback trials occurred in the context of a high probability of feedback occurrence. Thus, trial type interacts with prospective intentional binding effects based on a recent context, but not when based on an extended, less recent context. The strength of this interaction between trial type and context was 
The evolving sense of agency: Context recency and quality modulate the interaction between prospective and retrospective processes

1 negatively associated with psychosis-like experience trait scores. 2 Considering previous literature on the sense of agency (Moore, 2016; 3 Haggard, 2017; Bandura, 2018), we suggest that context-related effects 4 in our study are associated with prospective and attentional5 perceptual agency mechanisms, because the retrospective sense of 6 agency represents inferential, purely outcome-dependent processes.

Nevertheless, we also found a significant retrospective component represented by the difference in the perception of feedback vs. no-feedback trials, as indicated by the significance of the factor trial-type across all the trial windows. Feedback trials elicited stronger intentional binding, with a perceptual shift $(\Delta)$ closer to zero compared to no-feedback trials. Although this may appear in disagreement with findings of a nonsignificant retrospective sense of agency using the same paradigm (Voss et al., 2010), a small though statistically nonsignificant retrospective component was found in previous investigations (Di Plinio et al., 2019a; Di Plinio, Perrucci, \& Ebisch, submitted). Such a retrospective effect is consistent with,

18 and expands, current models of the sense of agency, showing its independence from narrow as well as extended contexts (Synofzik et al., 2008, 2013). We suggest that a significant retrospective effect in our study was detected by an accurate modeling of the response variable. This finding was made possible by considering the interaction between retrospective and prospective intentional binding effects, by including a relatively large number of participants, and by implementing a multilevel mixed-effects regression model.

Regarding prospective processes, previous studies have shown that a dynamic, evolving context conceivably influences the sense of agency through the dynamic modulation of predictive mechanisms (Wolpert et al., 2011; Clark, 2013) instantiated in sensorimotor and executive brain networks (Gallese \& Sinigaglia, 2010; Di Plinio et al., 2019b). As suggested by existing models of the sense of agency (Friston \& Kiebel, 2009; Friston, 2012; Thilakarathne \& Treur, 2015; Buhrmann \& Di Paolo, 2017; Legaspi \& Toyoizumi, 2019), these predictive mechanisms are putatively mediated by implicit sensorimotor 35 learning (McDougle et al., 2016; Kim et al., 2019). On the one hand, 36 we confirmed the existence of a classic, prospective sense of agency 
The evolving sense of agency: Context recency and quality modulate the interaction between prospective and retrospective processes

1 in our experiment (Haggard et al., 2002; Moore \& Haggard, 2008; Voss

2 et al., 2010; Synofzik et al., 2008, 2013). This context was

3 established by trials within a temporally extended context of action

4 feedback. In this case, intentional binding (and thus the sense of 5 agency) was increased with a higher probability of sensory

6 consequences. Interestingly, this contextual effect was equally

7 observable in feedback trials and no-feedback trials and can therefore

8 be considered independent of the actual action consequence.

On the other hand, the sense of agency based on a narrow context

0 of the online experience was influenced by the current trial type. In

11 fact, we found an interaction between context and trial-type within a

12 narrow context of action feedback: a high probability of tones in a

13 window of less than seven prior trials increased intentional binding

14 when the expected action feedback was missing, that is, in no-feedback

15 trials. This suggests that a recent, high-probability context of

16 agency generates a robust expectation and that a conflicting event

17 (missing feedback) increases intentional binding effects. We

18 hypothesize that such interaction may depend on both attentive and 19 perceptual-cognitive processes, as follows. First, a narrow high-tone-

20 probability context implies an increased rate of physical interactions

21 between the participant and the environment. This increases the

22 participant's engagement and externally oriented attention (Schooler

23 et al., 2011; Di Plinio et al., 2019a), possibly causing a shift of

24 the perception toward the expected tone (Correa et al., 2006; Burgess

25 et al., 2019). Second, the interaction between context and trial-type

26 may rely on perceptual Bayesian-like processes underlying sensorimotor

27 learning: when a strong prediction is generated, brain predictive

28 coding mechanisms enhance the expectation that the action consequence

29 is going to happen, and this expectancy might then be sustained over

30 time (De Ridder et al., 2014; Parr et al., 2019). Consequently, the

31 median expectation of the sensory consequence is delayed. In other

32 words, in the case of strongly expected missing feedback, the time

33 window during which the individual waits for feedback is stretched,

34 ultimately resulting in a shift of the temporal estimate toward the expected feedback. 
The evolving sense of agency: Context recency and quality modulate the interaction between prospective and retrospective processes

Notably, psychosis-like experience traits (F2) were associated with the strength of the interaction context:trial-type (i.e., the strength of the interaction of predictive and retrospective sense of

4 agency components) within a narrow context. In particular, psychosis-

5 like experience traits negatively correlated with intentional binding 6 effects when action feedback was lacking and when a narrow context 7 suggested a high probability for feedback. Thus, intentional binding 8 is modulated when the current trial type conflicts with the narrow 9 context in individuals with low levels of psychosis-like traits. This possibly favors a flexible sensorimotor learning based on recent and current evidence. By contrast, individuals with high levels of psychosis-like traits may need more time or more evidence to adapt to a dynamically evolving context. This finding agrees with previous 14 reports of both behavioral (Moore et al., 2011) and neural (Williams, et al., 2013; Giersch, et al., 2016) evidence aberrant habituation in schizophrenic patients. Previous studies have also found that deficiency of motor adaptation correlates with positive clinical symptoms in schizophrenia (Linney et al., 1998; Van Dael et al., 2006; Garety et al., 2011; Bansal et al., 2019). These effects are probably related to an impaired implicit learning of new rules or contexts (Frith et al., 2000; Blakemore et al., 2002; Ford \& Mathalon, 2005; Hauser et al., 2011; Martinelli et al., 2017) and may be exacerbated in situations of diminished environmental control, ambiguity, and unpredicted events (Moore et al., 2011; Gallagher \& Trigg, 2016, Di Plinio et al., 2019a, 2019b) .

To summarize, we maintain that psychosis-like traits are associated with less efficient sensorimotor predictions and a reduction in the adaptability of the subjective sense of agency to ongoing sensory experience. We suggest that previous findings of psychotic or schizotypal traits during the manipulation of the sense of agency (Voss et al., 2010; Hauser et al., 2011; Moore \& Fletcher, 2012; Martinelli et al., 2017; Di Plinio et al., 2019a) could be related to the interaction between prospective and retrospective

34 features. Contemplating such an effect in the Bayesian-like framework 35 highlighted above, we hypothesize that the expectation enhancement in 36 a narrow high-probability context is weakened in participants with 37 increased psychosis-like experiences because of aberrant sensorimotor 
The evolving sense of agency: Context recency and quality modulate the interaction between prospective and retrospective processes

1 adaptation to the external environment. Figure 6 provides a graphical 2 representation of this effect. Our findings trace a link between 3 personality traits and the emerging sense of agency, suggesting that 4 psychotic traits may undermine the updating of predictive sensorimotor

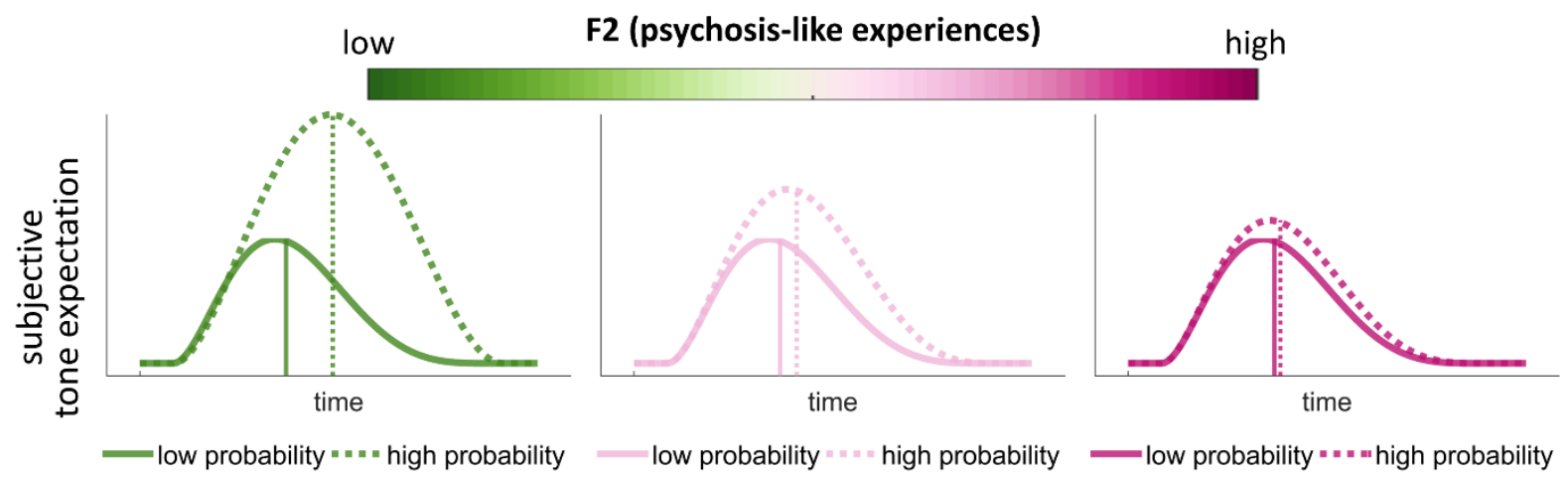

5

mechanisms also in healthy individuals.

\begin{abstract}
Figure 6. Bayesian interpretation of the effect of psychosis-like traits on intentional binding for narrow contexts. According to the model, in the case of missing feedback, the median expectation for that feedback is protracted over time within a narrow high-probability context (left panel, dashed line) but not within a narrow low-probability context of agency (left panel, continuous line). This implies a shift forward in the perceived timing of the action, represented by a greater intentional binding in our study. Instead, with increasing scores in psychotic traits (central and right panel), this expectation is no longer generated; the interaction effect is reduced as reflected by a decreased intentional binding for the narrow highprobability context, possibly as a consequence of impaired sensorimotor adaptation.
\end{abstract}

Our study has some limitations. For example, context-specific interactions between prospective and retrospective sense of agency were only assessed in the classic rotating clock experiment. However, recent studies have reported local and global temporal effects on implicit learning at both the neural and behavioral levels (Correa et al., 2006; Rolke \& Hofmann, 2007; Ball et al., 2018; Burgess et al., 2019), supporting the generalization of our findings across diverse sense of agency paradigms. Furthermore, we investigated implicit but not explicit (Haggard, 2017; Imaizumi \& Tanno, 2019) measures of the sense of agency, which are known to be at least partially divergent (Moore et al., 2012). Future studies may benefit from considering large samples to test time-dependent and context-dependent effects on 
The evolving sense of agency: Context recency and quality modulate the interaction between prospective and retrospective processes

1 both implicit and explicit agency dimensions, alongside classic run-

2 based approaches.

3

4 5. CONCLUSION

5 Our findings revealed that prospective mechanisms of the sense of 6 agency can be retrospectively influenced by the nature of the ongoing 7 event, suggesting that predictive mechanisms rely on multiple temporal 8 scales. In view of our findings, future cognitive models of agency 9 should integrate the dynamic interplay between internally generated 10 predictions and sensorimotor interactions to explain the processes 11 underlying the human sense of agency. Through the investigation of 12 interindividual differences in a large sample, our findings also have 13 clinical implications. We found that high scores in psychosis-like 14 experience traits in a non-clinical sample are associated with reduced 15 intentional binding effects in a narrow context of prospective sense 16 of agency, indicating weaker attentive-perceptual sensorimotor 17 adaptation. This lack of adaptation plausibly parallels the 18 difficulties in adapting to social contexts that accompany high 19 schizophrenic traits (de Bézenac, et al., 2015; van der Weiden, et 20 al., 2015), which possibly threatens the subjective sense of self as 21 a social agent during social interactions. Future studies are needed 22 to clarify whether such findings in individuals with high psychosis23 like traits are rooted in reduced habituation mechanisms, if they are 24 a consequence of a decreased exogenous attention, or if they reflect 25 abnormal predictive processes. Based on our findings, we suggest that 26 all these mechanisms may be critically involved. 
The evolving sense of agency: Context recency and quality modulate the interaction between prospective and retrospective processes

\begin{tabular}{|c|c|c|c|c|c|c|}
\hline \multirow{2}{*}{$\begin{array}{l}\text { TRIAL } \\
\text { WINDOW }\end{array}$} & \multicolumn{2}{|c|}{ Trial-type } & \multicolumn{2}{|c|}{ Context } & \multicolumn{2}{|c|}{ Interaction } \\
\hline & $\beta$ & pValue & $\beta$ & pValue & $\beta$ & pValue \\
\hline 1 & -5.9 & $<.001$ & -0.7 & .49 & 5.4 & $<.001$ \\
\hline 2 & -6.0 & $<.001$ & 0.0 & .96 & 3.5 & $<.001$ \\
\hline 3 & -6.2 & $<.001$ & 0.5 & .33 & 2.0 & .003 \\
\hline 4 & -5.9 & $<.001$ & 0.7 & .13 & 1.3 & .022 \\
\hline 5 & -5.9 & $<.001$ & 0.7 & .069 & 1.0 & .033 \\
\hline 6 & -5.6 & $<.001$ & 0.6 & .077 & 0.9 & .030 \\
\hline 7 & -5.8 & $<.001$ & 0.7 & .036 & 0.7 & .062 \\
\hline 8 & -5.8 & $<.001$ & 0.7 & .023 & 0.5 & .13 \\
\hline 9 & -5.8 & $<.001$ & 0.6 & .018 & 0.4 & .16 \\
\hline 10 & -5.9 & $<.001$ & 0.7 & .005 & 0.3 & .35 \\
\hline 11 & -5.9 & $<.001$ & 0.7 & .004 & 0.2 & .40 \\
\hline 12 & -5.9 & $<.001$ & 0.6 & .003 & 0.2 & .37 \\
\hline 13 & -5.7 & $<.001$ & 0.6 & .004 & 0.2 & .36 \\
\hline 14 & -5.8 & $<.001$ & 0.5 & .004 & 0.2 & .31 \\
\hline 15 & -5.7 & $<.001$ & 0.5 & .003 & 0.2 & .30 \\
\hline 16 & -5.8 & $<.001$ & 0.5 & .006 & 0.3 & .19 \\
\hline 17 & -5.8 & $<.001$ & 0.4 & .007 & 0.2 & .22 \\
\hline 18 & -5.8 & $<.001$ & 0.3 & .023 & 0.3 & .08 \\
\hline 19 & -5.7 & $<.001$ & 0.3 & .019 & 0.3 & .10 \\
\hline 20 & -5.7 & $<.001$ & 0.4 & .009 & 0.3 & .12 \\
\hline
\end{tabular}

2

3

4

5

6

7

Table I. Statistics of the trial-based analyses. Beta estimates and $p$ values for the three effects, i.e., trial-type, context, and the interaction context:trial-type. Significant results are highlighted in bold font. Non-significant results are shaded (threshold: $p<0.05$, uncorrected). 
The evolving sense of agency: Context recency and quality modulate the interaction between prospective and retrospective processes

\begin{tabular}{|c|c|c|c|c|c|}
\hline & F1 & F2 & F3 & F4 & F5 \\
\hline SPQ - INTERPERSONAL & .27 & .25 & -.39 & .84 & -.02 \\
\hline SPQ-DISORGANIZED & .32 & .36 & .17 & .57 & -.16 \\
\hline SPQ-COGNITIVE & .20 & .68 & -.15 & .39 & -.04 \\
\hline CAPE-POSITIVE & .05 & .98 & -.12 & .10 & -.07 \\
\hline CAPE-NEGATIVE & .34 & .44 & -.22 & .34 & -.30 \\
\hline CAPE-DEPRESSIVE & .79 & .37 & .01 & .16 & -.18 \\
\hline STAI-TRAIT & .81 & .11 & -.13 & .16 & -.19 \\
\hline IUS-INHIBITORY & .47 & .03 & -.34 & .26 & -.26 \\
\hline IUS-PROSPECTIVE & .26 & .20 & -.48 & .02 & .09 \\
\hline BEQ-EMOT.STABILITY & -.69 & -.01 & .29 & -.16 & -.07 \\
\hline BEQ-EXTRAVERSION & -.36 & .26 & .49 & -.21 & .15 \\
\hline BFQ-AGREEABLENESS & .03 & -.09 & .50 & -.12 & .11 \\
\hline BFQ-OPENNESS & -.21 & -.08 & .61 & .06 & .33 \\
\hline BFQ-CONSCIENTIOUSNESS & -.15 & -.09 & .20 & -.09 & .78 \\
\hline
\end{tabular}

1

2 Table II. Questionnaire contributions to psychometric factors. Bold

3 values indicate scales with a large contribution to a specific factor 4 (eigenvalue $>0.40)$. Shading indicates scales with a small 5 contribution to a specific factor (eigenvalue $<0.30$ ). $\mathrm{SPQ}=$ 6 Schizotypal Personality Questionnaire; CAPE = Community Assessment of 7 Psychic Experience; STAI = State-Trait Anxiety Inventory; IUS = 8 Intolerance of Uncertainty Scale; BFQ = Big Five Questionnaire. 9 
The evolving sense of agency: Context recency and quality modulate the interaction between prospective and retrospective processes

1

2

4

5

\section{REFERENCES}

Asai, T, Sugimori, E, Tanno, Y (2008). Schizotypal personality traits and prediction of one's own movements in motor control: What causes an abnormal sense of agency? Consciousness and Cognition, 17 (4):1131-1142.

Asai, T, Sugimori, E, Bando, N, Tanno, Y (2011). The hierarchic structure in schizotypy and the five-factor model of personality. Psychiatry Research, 185(12), 78-83.

Ball, F, Fuehrmann, F, Stratil, F, Noesselt, T (2018). Phasic and sustained interactions of multisensory interplay and temporal expectation. Scientific Reports, $8(1), 1-12$.

Bandura, A (2018). Toward a psychology of human agency: Pathways and reflections. Perspectives in Psychological Science. 13(2), 130-136.

Bansal, S, Murthy, KG, Fitzgerald, J, Schwartz, BL, Joiner, WM (2019). Reduced transfer of visuomotor adaptation is associated with aberrant sense of agency in schizophrenia. Neuroscience, 413, 108-122.

Berberian, B, Sarrazin, JC, Le Blaye, P, Haggard, P (2012). Automation technology and sense of control: a window on human agency. PLoS One, 7 (3):e34075.

Blakemore, SJ, Wolpert, DM, Frith, CD (2002). Abnormalities in the awareness of action. Trends in Cognitive Sciences, 6, 237-242.

Bohrani, K, Beck, B, Haggard, P (2017). Choosing, doing, and controlling: Implicit sense of agency over somatosensory events. Psychological Science, 28(7), 882893 .

Buhrmann, T, Di Paolo, E (2017). The sense of agency - A phenomenological consequence of enacting sensorimotor schemes. Phenomenology and the Cognitive Sciences, $16(2), 207-236$.

Burgess, JD, Major, BP, MCNeel, C, Clark, GM, Lum, JAG, Enticoot, PG (2019). Learning to expect: Predicting sounds during movement is related to sensorimotor association during listening. Frontiers in Human Neuroscience, 13(215), $1-14$.

Caprara, GV, Barbaranelli, C, Borgogni, L, Perugini, M (1993). The "Big Five Questionnaire": A new questionnaire to assess the five factor model. Personality and Individual Differences, $15(3), 281-288$.

Carleton, RN, Norton, MA, Asmundson, GJG (2007). Fearing the unknown: A short version of the intolerance of uncertainty scale. Journal of Anxiety Disorders, $21,105-117$.

Clark, A (2013). Whatever next? Predictive brains, situated agents, and the future of cognitive science. Behavioral and Brain Sciences, 36(3), $181-204$.

Correa, À, Lupiànez, J, Madrid, E, Tudela, P (2006). Temporal attention enhances early visual processing: A review and new evidence from event-related potentials. Brain Research, 1076, 116-128.

Costa, PT, McCrae, RR (1985). The NEO personality inventory manual. Odessa, FL: Psychological Assessment Resources.

Desantis, A, Waszak, F, Moutsopoulou, K, Haggard, P (2016). How action outcome structures time: About the perceived temporal order of action and predicted outcomes. Cognition, 146, 100-109.

de Bézenac, CE, Sluming, V, O'Sullivan, N, Corcoran, R (2015). Ambiguity between self and other: Individual differences in action attribution. Consciousness and cognition, 35, 1-15.

De Ridder, D, Vanneste, S, Freeman, W (2014). The Bayesian brain: Phantom percepts resolve sensory uncertainty. Neuroscience and Biobehavioral Reviews, 44, 4-15.

de Winter, JCF, Dodou, D, Wiering, PA (2009). Exploratory factor analysis within small sample sizes. Multivariate Behavioral Research, 44(2), $147-181$.

Di Plinio, S, Arnò, S, Perrucci, MG, Ebisch, SJH (2019a). Environmental control and psychosis-relevant traits modulate the prospective sense of agency in nonclinical individuals. Consciousness and Cognition, 73, 102776. 
The evolving sense of agency: Context recency and quality modulate the interaction between prospective and retrospective processes

Di Plinio, S, Perrucci, MG, Aleman, A, Ebisch, SJH (2019b). I am Me: Brain systems integrate and segregate to establish a multidimensional sense of self. NeuroImage, 205, 116284 .

Di Plinio, S, Perrucci, MG, Ebisch, SJH (2019). The prospective sense of agency is rooted in local and global properties of intrinsic functional brain networks. Submitted.

Dogge, M, Custers, R, Gayet, S, Hoijtink, H, Aarts, H (2019). Perception of actionoutcome is shaped by life-long and contextual expectations. Scientific Reports, $9(1), 5225$.

Ford, JM, Mathalon, DH (2005). Corollary discharge dysfunction in schizophrenia: Can it explain auditory hallucinations? International Journal of Psychophysiology, 58, 179-189.

Fossati, A, Raine, A, Carretta, I, Leonardi, B, Maffei, C (2003). The three-factor model of schizotypal personality: Invariance across age and gender. Personality and Individual Differences, 35(5), 1007-1019.

Friston, K, Kiebel, S (2009). Predictive coding under the free-energy principle. Philosophical Transactions of the Royal Society B: Biological Sciences, $364: 1211-1221$.

Friston, K (2012). Prediction, perception and agency. International Journal of Psychophysiology, $83(2), 248-252$.

Frith, CD, Blakemore, S, Wolpert, DM (2000). Explaining the symptom of schizophrenia: Abnormalities in the awareness of action. Brain Research Reviews, $31,357-363$.

Gallagher, S, Trigg, D (2016). Agency and Anxiety: Delusions of control and loss of control in Schizophrenia and Agoraphobia. Frontiers in Human Neuroscience, 10, 459 .

Gallese, V, Sinigaglia, G (2010). The bodily self as power for action. Neuropsychologia, $48(3), 746-755$.

Garety, P, Freeman, D, Jolley, S, Ross, K, Waller, H, Dunn, G (2011). Jumping to conclusions: the psychology of delusional reasoning, Advances in Psychiatric Treatment, $17,332-339$.

Giersch, A, Lalanne, L, Isope, P (2016). Implicit timing as the missing link between neurobiological and self disorders in schizophrenia? Frontiers in Human Neuroscience, 10, 103 .

Haggard, P, Clark, S, Kalogeras, J (2002). Voluntary action and conscious awareness. Nature Neuroscience, $5(4): 382$.

Haggard, P (2017). Sense of agency in the human brain. Nature Reviews Neursocience, $18(4): 196$.

Hauser, M, Knoblich, G, Repp, BH, Lautenschlager, M, Gallinat, J, Heinz, A, Voss, M (2011). Altered sense of agency in schizophrenia and the putative psychotic prodrome. Psychiatry Research, $186(2-3), 170-176$.

Horn, JL (1965). A rationale and test for the number of factors in factor analysis. Psychometrica, $30(2), 179-185$.

Imaizumi, S, Tanno, Y (2019). Intentional binding coincides with explicit sense of agency. Consciousness and Cognition, 67:1-15.

Jeannerod, M (2009). The sense of agency and its disturbances in schizophrenia: A reappraisal. Experimental Brain Research, $192(3), 527$.

Kim, HE, Parvin, DE, Ivry, RB (2019). The influence of task outcome on implicit motor learning. Elife, 8, e39882.

Konings, M, Bak, M, Hanssen, M, van Os, J, Krabbendam, L (2006). Validity and reliability of the CAPE: A self-report instrument for the measurement of psychotic experiences in the general population. Acta Psychiatrica Scandinavica, $114(1), 55-61$.

Korth, B, Tucker, LR (1976). Procrustes matching by congruence coefficients. Psychometrika, 41(4), 531-535. 
The evolving sense of agency: Context recency and quality modulate the interaction between prospective and retrospective processes

Kumar, D, Srinivasan, N (2013). Hierarchical control and sense of agency: Differential effects of control on implicit and explicit measures of agency. Proceedings of the Annual Meeting of the Cognitive Science Society. 35(35).

Ledesma, RD, Valero-Mora, P (2007). Determining the number of factors to retain in EFA: An easy-to-use computer program for carrying out parallel analysis. Practical assessment, Research \& Evaluation, $12(2), 2-11$.

Legaspi, R, Toyoizumi, T (2019), A bayesian psychophysics model of sense of agency. Nature Communications, 10 (4250).

Linney, YM, Peters, ER, Ayton, P (1998). Reasoning biases in delusion-prone individuals. British Journal of Clinical Psychology, 37, 285-302.

Lorenzo-Seva, U, ten Berge, JMF (2006). Tucker's congruence coefficient as a meaningful index of factor similarity. Methodology: European Journal of Research Methods for the Behavioral and Social Sciences, 2 (2), 57-64.

Martinelli, C, Rigoli, F, Shergill, SS (2017). Aberrant force processing in schizophrenia. Schizoprenia Bulletin, 43, 417-424.

McDougle, SD, Ivry, RB, Taylor, JA (2016. Taking aim at the cognitive side of learning in sensorimotor adaptation tasks. Trends in Cognitive Sciences, 20(7), $535-544$.

Moore, JW, Haggard, P (2008). Awareness of action: Inference and prediction. Consciousness and Cognition. 17(1), 136-144.

Moore, JW, Dickinson, A, Fletcher, PC (2011). Sense of agency, associative learning, and schizotypy. Consciousness and Cognition, 20, 792-900.

Moore, JW, Middleton, D, Haggard, P, Fletcher, PC (2012). Exploring implicit and explicit aspects of sense of agency. Consciousness and Cognition, 21(4), 17481753 .

Moore, JW, Fletcher, PC (2012). Sense of agency in health and disease: A review of cue integration approaches. Consciousness and Cognition, 21, 59-68.

Moore, JW (2016). What is the sense of agency and why does it matter? Frontiers in Psychology, 7, 1272 .

Parr, T, Corcoran, AW, Friston, KJ, Hohwy, J (2019). Perceptual awareness and active inference. Neuroscience of Consciousness, $2019(1)$, niz012.

Raine, A (1991). The SPQ: A scale for the assessment of schizotypal personality based on DSM-III-R criteria. Schizophrenia Bulletin, 17 (4), 555-564.

Rolke, B, Hofmann, P (2007). Temporal uncertainty degrades perceptual processing. Psychonomic Bulletin and Review, 14(3), 522-526.

Schneider, W, Eschman, A, Zuccolotto, A (2012). E-Prime 2.0 Reference Guide Manual. Pittsburgh: Psychology Software Tools Inc.

Schooler, JW, Mrazek, MD, Franklin, MS, Baird, B, Mooneyham, BW, Zedelius, C, Broadway, JM (2014). The middle way. Psychology of Learning and Motivation (Elsevier), Burlington: Academic Press, 1-33.

Sidarus, N, Vuorre, M, Haggard, P (2017). Integrating prospective and retrospective cues to the sense of agency: A multi-study investigation. Neuroscience of consciousness, 1, nix012.

Spielberger, CD, Gorssuch, RL, Lushene, PR, Vagg, PR, Jacobs, GA (1983). Manual for the state-trait anxiety inventory. Consulting Psychologists Press.

Stark-Inbar, A, Raza, M, Taylor, JA, Ivry, PB (2017). Individual differences in implicit motor learning: Task specificity in sensorimotor adaptation and sequence learning. Journal of Neurophysiology, $117(1), 412-428$.

Synofzik, M, Vosgerau, G, Newen, A (2008). Beyond the comparator model: A multifactorial two-step account of agency. Consciousness and Cognition, 17(1), 219-239.

Synofzik, M, Vosgerau, G, Voss, M (2013). The experience of agency: An interplay between prediction and postdiction. Frontiers in Psychology, 4, 127. 
The evolving sense of agency: Context recency and quality modulate the interaction between prospective and retrospective processes

Taylor, JA, Krakauer, JW, Ivry, PB (2014). Explicit and implicit contributions to learning in a sensorimotor adaptation task. Journal of Neuroscience, 34 (8), 3023.3032 .

Thilakarathne, DJ, Treur, J (2015). Computational cognitive modelling of action awareness: Prior and retrospective. Brain Informatics, 2(2), 77-106.

Van Dael, F, Versmissn, D, Janssen, I, Myin-Germeys, I, van Os, J, Krabbendam, L (2006). Data gathering: biased in psychosis? Schizophrenia Bulletin, 32, 341351 .

van der Weiden, A, Prikken, M, van Haren, NE (2015). Self-other integration and distinction in schizophrenia: A theoretical analysis and a review of the evidence. Neuroscience and Biobehavioral Reviews, 57, 220-237.

Van Kemenade, BM, Arikan, BE, Kircher, T, Straube, B (2016). Predicting the sensory consequences of one's own action: First evidence for multisensory facilitation. Attention Perception Psychophysics, 78(8), 2515-2526.

Voss, M, Moore, J, Hauser, M, Gallinat, J, Heinz, A, Haggard, P (2010). Altered awareness of action in schizophrenia: a specific deficit in predicting action consequences. Brain, 133(10), 3104-3112.

Wen, W (2019). Does delay in feedback diminish the sense of agency? A review. Consciousness and Cognition, 73(192759), 1-9.

Williams, LE, Blackford, JU, Luksik, A, Gauthier, I, Heckers, S (2013). Reduced habituation in patients with schizophrenia. Schizophrenia Research, 151(0), 124132 .

Wolpert, DM, Diedrichsen, J, Flanagan, J R (2011). Principles of sensorimotor learning. Nature Reviews Neuroscience, $12(12), 739-751$. 